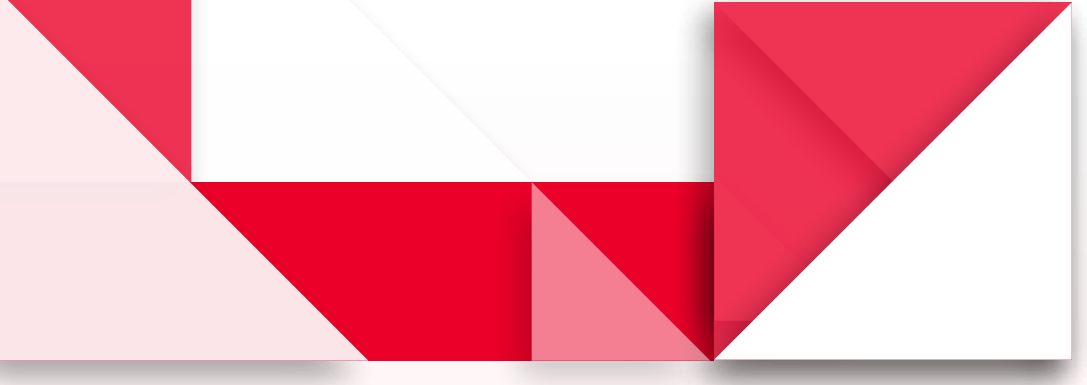

\title{
La enseñanza ambiental \\ 3 como propuesta de formación integral
}
- The environmental education as a proposal of integral formation

- O ensino ambiental como proposta de formação integral

\section{David Esteban Lastra Romero Ángela Gineth Ramírez Pachón'}

\footnotetext{
1 Licenciados en Biología. Universidad Pedagógica Nacional-delastrar@gmail.com, angirapa17@hotmail.com
}

\section{Resumen}

Este artículo presenta los resultados obtenidos de una investigación sobre la construcción del Seminario de Enseñanza Ambiental para los maestros en formación de la Facultad de Ciencia y Tecnología de la Universidad Pedagógica Nacional, para lo cual se tomó el enfoque critico social y el modelo investigativo cualitativo como ejes estructurantes en la metodología. Se remite a la teoría de la complejidad según Morin y al de la complejidad ambiental según Leff, como base para cuestionar los modelos establecidos en la educación ambiental y fundamentar la propuesta de enseñanza ambiental, teniendo en cuenta publicaciones, trabajos de grado, líneas de investigación, programas académicos y seminarios orientados hacia el abordaje de la educación ambiental encontrados en la Facultad de Ciencia y Tecnología de la Universidad Pedagógica Nacional.

\section{Abstract}

This article presents the results of a study on the construction of the environmental education seminar for pre service teachers in the Science and Technology Faculty at Universidad Pedagógica Nacional. The critical social approach and the qualitative research model were considered as constructs in the methodology. It is based on the theory of complexity according to Morin and environmental complexity according to Leff in order to question the established models in environmental education and support the environmental education proposal, taking into account publications, project work, research, academic programs and seminars regarding environmental education, which can be found in the Science and Technology Faculty at

\section{Keywords:}

environmental education, Integral formation, Seminar, Environment, Environmental problematic, complex thinking. Universidad Pedagógica Nacional. 


\section{Resumo}

Este artigo apresenta os resultados obtidos em uma pesquisa sobre a construção do Seminário de "Enseñanza Ambiental" para os professores em formação da "Facultad de Ciencia y Tecnología de la Universidad Pedagógica Nacional", utilizando o enfoque crítico social e o modelo investigativo qualitativo como eixos estruturantes da metodologia. Remete-se à teoria da complexidade, conforme Morin, e a complexidade ambiental, conforme Leff, como base para questionar os modelos estabelecidos na educação ambiental e fundamentar a proposta de ensino ambiental, considerando publicações, trabalhos de conclusão de curso de graduação, linhas de pesquisa, programas acadêmicos e Seminários orientados para a abordagem da educação ambiental no âmbito da "Facultad de Ciencia y Tecnología de la Universidad Pedagógica Nacional".

\section{Palavras-chave:}

ensino Ambiental, Formação Integral, Seminário, Educação Ambiental, Ambiente, Problemática Ambiental, Pensamento Complexo. 


\section{Problema de investigación}

Con la abundante información acerca de la condición actual del mundo resulta imposible negar la crisis ambiental que compromete el futuro de la humanidad y de toda la vida sobre el planeta Tierra. Esto se puede detectar en la extensa lista de situaciones que se reportan a diario sobre aspectos como la cantidad de basura que se produce, la contaminación de las fuentes de agua, el cambio climático global, entre otros. La percepción de dichas situaciones conduce a la búsqueda de alternativas para detener $\mathrm{o}$, al menos, disminuir el impacto de las actividades humanas sobre el entorno, lo que se hace principalmente con estudios y trabajos desde el campo académico.

La mayoría de las soluciones que se proponen a los problemas ambientales son alternativas que están enmarcadas en lo que Eschenhagen (2009) denomina la racionalidad instrumental y económica, dado que deben presentar resultados inmediatos con respecto a la disminución de un problema particular y además deben ser viables económicamente. La situación anterior puede atribuirse a que las causas por las cuales se genera la degradación del ambiente no son claras y dependen del esquema interpretativo desde el que se aborden los problemas; en otras palabras, el origen que se le atribuya a la problemática ambiental dependerá de la manera como se piensa y del marco conceptual desde el cual se aborde.

Así, es necesario tener claridad sobre las causas profundas de la cuestión ambiental para pensar alternativas que permitan un acercamiento a la compleja dinámica de este tema. A este respecto, Leff (2004) plantea que "la problemática ambiental emerge como una crisis de la racionalidad del mundo moderno" que se caracteriza por ser científica y económica. Es dicha racionalidad la que legitima la destrucción de la naturaleza y la degradación de la humanidad en nombre del progreso. El mismo autor sugiere también que "la crisis ambiental es la crisis del efecto del conocimiento sobre el mundo", refiriéndose particularmente a la ciencia como forma de conocer dominante que fragmenta el mundo y lo desestructura.

Frente a dicha crisis surge la educación ambiental como propuesta que cuestiona la racionalidad económica haciendo evidente la necesidad de un nuevo modelo de desarrollo y sobre todo, una nueva forma de pensar. No obstante, con el paso del tiempo y atendiendo a los intereses de la globalización, tal propuesta comienza a perder su sentido crítico con respecto a la racionalidad económica y se enfoca en la educación para el desarrollo sostenible; es decir, el problema ya no es cuestionar la racionalidad moderna sino cómo hacerla "sostenible".

Desde esta perspectiva se incorpora la educación ambiental al sistema educativo colombiano y se plantea como una estrategia denominada Proyecto Ambiental Escolar (PRAE) el cual debe ser aplicado y desarrollado por los maestros de las instituciones educativas. Sin embargo, los maestros no tuvieron la oportunidad de pensar detenidamente si un proyecto es la mejor manera de tratar la problemática ambiental ni de establecer otra estrategia para abordarlas. Por si fuera poco, la responsabilidad de dichos proyectos no recae sobre toda la institución educativa, pues, por lógica, quienes deben hacerse cargo de un proyecto que se denomina ambiental son aquellos que conocen los temas asociados al ambiente, es decir los profesores de ciencias naturales. Esta situación, además de mostrar cómo el conocimiento está fragmentado, presenta una visión del ambiente como problema que se puede solucionar con un proyecto y reduce la labor docente y la educación misma a la 
aplicación de estrategias, como si se tratara de protocolos para obtener un producto determinado.

A partir de lo anterior, la educación ambiental en los programas de formación de maestros de ciencias, más que proporcionar recetas para solucionar problemas, debería comenzar por cuestionar todo aquello que se ha establecido en los marcos metodológicos y teóricos que orientan su desarrollo, para dar la oportunidad de pensar y proponer diferentes formas de abordar los problemas ambientales. Por esta razón, se considera pertinente la elaboración de una propuesta de enseñanza ambiental cuyo fundamento sea justamente la reflexión sobre las diferentes maneras en que se puede pensar y desarrollar lo ambiental desde los diferentes campos de conocimiento.

\section{Marco legal y teórico}

El discurso sobre el ambiente aparece en la historia por las diferentes tensiones que se generan en las relaciones de la humanidad con la naturaleza, particularmente cuando implican el deterioro de los recursos naturales y la contaminación de los ecosistemas como producto de las actividades humanas en el mundo. Como resultado de esta situación, emergen movimientos ecologistas, ambientalistas y conservacionistas que cuestionan las acciones de la sociedad sumergida en la visión económica y productiva del mundo (Gutiérrez, 1995a). No obstante, este tema comienza a ser relevante a nivel mundial solamente cuando el mismo modelo económico se ve amenazado por la sobrepoblación y la escasez de recursos.

La constatación de una naturaleza que está alcanzando sus límites en cuanto a extracción y producción, propicia la preocupación por el ambiente, no solo por parte de grupos académicos o independientes sino a nivel gubernamental, político y económico. Por esto, lo ambiental comienza a ser discutido en reuniones internacionales o cumbres en las cuales se establece que la problemática ambiental se encuentra íntimamente asociada con la dinámica de las sociedades humanas y que existe una estrecha relación entre la destrucción ambiental y el orden económico internacional, por tanto, este tema debe considerarse como un asunto global (Eschenhagen, 2009; Maya, 2009; Gaudiano, 2001; Gutiérrez, 1995b).

En dichos encuentros la educación se reconoce como elemento de "concienciación" mundial sobre el tema ambiental lo que conduce al surgimiento de la educación ambiental (EA) en 1975 con la creación del Programa Internacional de Educación Ambiental (PIEA) apoyado en varias reuniones como la de Tbilisi en la cual se propone incorporarla a los sistemas educativos de los países (Gaudiano, 2001 ; Rojas, 2006).

Aunque la emergencia de la EA es fundamental en el abordaje de la problemática a nivel mundial, es necesario considerar que una propuesta de aplicación internacional puede desconocer dinámicas particulares de diferentes lugares en el mundo puesto que el contexto natural, social, cultural y económico varía de 
un país a otro. Por tal razón, en América Latina se llevan a cabo reuniones alternas para discutir la situación de los países neotropicales, atendiendo a que tienen una mayor diversidad biológica en comparación con los países nórdicos y unos procesos sociales diferentes en cuanto al deterioro del ambiente (Gaudiano, 2001; Tréllez, 2006; Calvo y Pérez, 2006). A pesar de esto, las aplicaciones concretas en materia ambiental obedecen a intereses internacionales en pro de mantener el modelo económico, de allí el surgimiento del desarrollo sostenible y de la educación para el desarrollo sostenible, en donde la cuestión no es buscar alternativas al modelo económico sino cómo lograr un mejor provecho de los recursos y que sean suficientes para abastecer a las generaciones futuras.

En medio de este contexto, la EA llega a Colombia con la Constitución de 1991 y la Ley General de Educación 115 de 1994, en las que se introduce como disciplina obligatoria en el currículo escolar (Eschenhagen, 1998; SINA, 2002), a través del Proyecto Ambiental Escolar (PRAE). Sin embargo, el este último llega como una norma a las instituciones educativas y como un elemento de control sobre lo ambiental en el que se evidencia la fragmentación del problema y del conocimiento en general, pues quienes asumen tal proyecto habitualmente son los maestros de ciencias naturales, particularmente los de biología y química, sugiriendo que ellos son quienes deben abordar y solucionar los problemas asociados al ambiente en el ámbito educativo. De allí que se considere necesaria una formación ambiental diferente que no solo se dirija a maestros de ciencias sino a profesionales de todas las áreas dado que independientemente de los gustos o las disciplinas que escojan los sujetos, hay un una relación y un impacto sobre el ambiente que se debe pensar y reflexionar.

Por otra parte, en las discusiones y construcciones teóricas no hay claridad frente a lo que es el ambiente, la Educación Ambiental y la problemática ambiental, puesto que se han planteado y replanteado muchas definiciones desde el momento en que comenzó a debatirse el tema. Esto dificulta aún más el abordaje de lo ambiental en la escuela y dilata las propuestas educativas en activismos que no tienen un horizonte claro. Con tal abundancia de información no es posible unificar al ambiente en un solo concepto, pero sí se puede categorizar en tendencias; lo mismo ocurre con la EA y con la problemática ambiental (Sauvé, 1994; Eschenhagen, 2009). En esta investigación se toman en cuenta las categorías para estos conceptos de acuerdo con los autores relacionados en la tabla 1 .

A partir de lo anterior, al tratar el tema ambiental a través de un seminario en el campo de la educación superior, particularmente en la Facultad de Ciencia y Tecnología de la Universidad Pedagógica Nacional, se debe tener en cuenta que el humano actúa en el mundo a través de las formas de pensar y que la problemática ambiental, más allá de ser ecológica, es "una crisis del pensamiento y del entendimiento [...] de la forma en que la civilización occidental ha comprendido el ser, a los entes y las cosas" (Leff, 2007). Para tal fin, es necesario tratar este tema desde una perspectiva que permita preguntar por las causas últimas del problema ambiental, que no busque soluciones sino que problematice aquello que es un problema para comprender la complejidad del mundo bajo la influencia del conocimiento (Leff, 2007; Morin, 2007). 
Tabla 1. Determinación de categorías y subcategorías de acuerdo con los planteamientos teóricos de Eschenhagen (2009) para ambiente, Caride y Meira (2001) para educación ambiental y para problemática ambiental Eschenhagen (2009), Caride y Meira (2001) y Leff (2004).

\begin{tabular}{l|l}
\multicolumn{1}{c}{ Concepto } & \multicolumn{1}{c}{ Categoría o tendencia } \\
\hline \multirow{2}{*}{ Ambiente } & Ambiente como objeto. \\
\cline { 2 - 2 } & Ambiente como sistema. \\
\cline { 2 - 2 } & Ambiente como critica a la visión de mundo moderno. \\
\hline \multirow{2}{*}{ Educación ambiental } & La educación ambiental como acción tecnológica y ciencia aplicada. \\
\cline { 2 - 2 } & La educación ambiental como práctica social crítica. \\
\hline \multirow{2}{*}{$\begin{array}{l}\text { Problemática } \\
\text { ambiental }\end{array}$} & Como crisis del efecto del conocimiento sobre el mundo. \\
\cline { 2 - 2 } & Como construcción social. \\
\cline { 2 - 2 } & Algo para conocer, analizar y solucionar problemas ambientales. \\
\hline
\end{tabular}

\section{Metodología de la investigación}

La investigación se desarrolla bajo la perspectiva de la investigación cualitativa descrita desde tres fases, la indagación, caracterización y el diseño del seminario de enseñanza ambiental para los maestros en formación de la Facultad de Ciencia y Tecnología.

La fase de indagación se enfoca en la revisión, análisis de documentos y discursos que dieron a conocer las tendencias más frecuentes que frente al ambiente, educación ambiental y problemática ambiental se han desarrollado en la Facultad de Ciencia y Tecnología. Para ello se plantea una etapa A, enfocada en el análisis de texto como publicaciones, trabajos de grado y aquellos escritos que fundamentan las líneas de investigación.

La etapa B o análisis de discurso en donde se reconoce la postura académica o la forma como se entiende el ambiente, la educación ambiental y la problemática ambiental, la postura investigativa asociada a cómo se investiga lo ambiental y, por último, la metodológica o las propuestas que se han implementado o desarrollado en torno al tema ambiental; posturas que de una $u$ otra forma constituyen el discurso del maestro.

Se toma como instrumento etnográfico entrevistas no directivas aplicadas a directivos como el Decano de la Facultad de Ciencia y Tecnología, seguido de los coordinadores de programa así como de los coordinadores de las líneas de investigación. Por último a los maestros que propusieron los programas académicos de educación ambiental en el nivel de pregrado desde el año 2008 hasta 2011 , junto con un grupo de estudiantes (tres por periodo académico) que han tomado estos cursos. Por último, durante un mes y medio se realizan observaciones a los seminarios que sobre educación ambiental se ofrecieron en la Facultad de Ciencia 
y Tecnología en el segundo periodo académico de 2011.

La fase de caracterización, para la cual se desarrolló el análisis de contenido a los programas académicos, propuestos para los seminarios de educación ambiental desarrollados desde 2008 hasta 2011 . De este modo se seleccionan las categorías de investigación, tales como, objetivos a desarrollar dentro del curso, los cuales reflejan el interés del maestro, conceptos y tendencias asociados al pensamiento ambiental; las estrategias metodológicas como herramientas que permiten el cumplimiento de los objetivos; las unidades temáticas o temas donde se reflejaron los planteamientos teóricos abordados en el curso y por los cuales se fundamenta el maestro. Por último, se toma la selección de textos, dado que las fuentes bibliográficas identificadas señalaron la pertinencia de un estudio histórico, social y educativo en el desarrollo de un seminario de educación ambiental para la formación de maestros.

Finalmente, la fase de diseño se materializa el seminario, el cual se centra en el cuestionamiento de la visión del mundo moderno como causa central de la dinámica de la educación ambiental, así como el cambio en las formas de conocimiento que dominan actualmente y desvirtúan la formación integral del profesorado. Para el diseño no se tienen en cuenta referentes teóricos con respecto a la estructura del mismo, dado que esto se define desde los objetivos que pretenda el profesor desarrollador; no obstante se demarcan parámetros generales como las posturas de los investigadores, los objetivos del programa, las temáticas abordadas durante el seminario junto con la metodología y la descripción de las 16 sesiones orientadas desde cuestionamientos que dinamizarán y permitirán el debate y la construcción del discurso del maestro en formación.

\section{Resultados y análisis}

\section{Fase de indagación: etapa A}

Los primeros resultados, surgen de la etapa A de la fase de indagación, en la que se analizaron las publicaciones al interior de la Facultad de Ciencia y Tecnología, dentro de las cuales se destacan la revista Tecné Epistemé y Didaxis (TED), Prácticas Pedagógicas del Departamento de Química (PPDQ), y Bio-grafía en versión virtual. En la tabla 2 se identifican las tendencias de ambiente, educación ambiental y problemática ambiental para cada una de las revistas de la Facultad de Ciencia y Tecnología. 
Tabla 2. Resultados del análisis de contenido en la fase de indagación A. Identificación de las tendencias asociadas al ambiente, la Educación Ambiental y la problemática ambiental. En la tabla solo se relacionan aquellas publicaciones que aportaron información suficiente para categorizarlas, por consiguiente hubo documentos en los cuales no fue posible realizar este proceso. Convenciones: EA: Educación Ambiental DBI: Departamento de Biología; DQI: Departamento de Química; DEC: Departamento de Electrónica.

\begin{tabular}{|c|c|c|c|c|c|c|c|c|c|}
\hline \multirow{3}{*}{ Docume } & 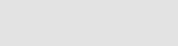 & \multicolumn{8}{|c|}{ Categorías } \\
\hline & 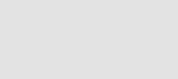 & \multicolumn{3}{|c|}{ AMBIENTE } & \multicolumn{3}{|c|}{ EDUCACION AMBIENTAL } & \multicolumn{2}{|c|}{$\begin{array}{c}\text { PROBLEMÁTICA } \\
\text { AMBIENTAL }\end{array}$} \\
\hline & tos revisados & & & $\frac{0}{0}$ & 흥 & : & $\overline{\overline{0}}$ 욤 & $\overline{\frac{0}{U}}$ & ì \\
\hline \multirow{3}{*}{$\begin{array}{l}\mathscr{U} \\
\stackrel{0}{0} \\
. \frac{O}{U} \\
\frac{0}{0} \\
\frac{.0}{0} \\
\frac{0}{D}\end{array}$} & TED (9) & 1 & 1 & & 5 & 1 & 1 & 1 & \\
\hline & Bio-grafía (8) & & & & 2 & 1 & & 1 & \\
\hline & PPDQ (3) & 2 & & & 2 & & & & 3 \\
\hline \multirow{3}{*}{ 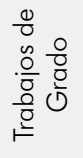 } & DBI (27) & 2 & 13 & 2 & 19 & 3 & & & 2 \\
\hline & DQI (15) & & 5 & & 12 & 2 & & & 5 \\
\hline & $\operatorname{DEC}(1)$ & & & & & & & & 1 \\
\hline \multirow{2}{*}{ 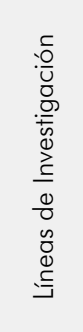 } & $\begin{array}{l}\text { DBI. EA en } \\
\text { el contexto } \\
\text { educativo } \\
\text { colombiano }\end{array}$ & & & $x$ & $x$ & & & & \\
\hline & $\begin{array}{l}\text { DQUI. } \\
\text { Incorporación } \\
\text { de la EA al } \\
\text { Currículo de } \\
\text { Ciencias }\end{array}$ & & $x$ & & $x$ & & & & $x$ \\
\hline
\end{tabular}

Se analizan 20 artículos, 28 trabajos de grado y 2 documentos que orientan las líneas de investigación que abordan la educación ambiental en la formación del profesor. Es preciso aclarar que tanto las publicaciones como las tesis están relacionadas con el abordaje y desarrollo de la Educación Ambiental; sin embargo, ninguno se asoció al diseño de propuestas curriculares, planes de estudio, o programas académicos de educación ambiental dirigidos a los diferentes niveles educativos ni a los departamentos que componen la Facultad de Ciencia y Tecnología.

En términos generales, el resultado de esta categorización muestra que las tendencias predominantes se asocian a la visión de ambiente como sistema (Eschenhagen, 2009), educación ambiental como acción tecnológica y ciencia aplicada (Caride y Meira, 2001) y problemática ambiental como algo para conocer, analizar y solucionar (Eschenhagen, 2009) (tabla 2). En este sentido, se puede afirmar que en la Facultad de Ciencia y Tecnología son escasos aquellos 
documentos propuestos desde un marco diferente al establecido internacionalmente para estas categorías y, por tanto, las concepciones asociadas se ubican en tendencias que refieren a la forma clásica en que se ha abordado lo ambiental en la escuela, es decir, como algo que se debe estudiar, comprender y solucionar a través de la formación científica.

Además, pocos documentos plantean una crítica a los fundamentos políticos y económicos de la Educación Ambiental; por el contrario, señalan la necesidad de ver el ambiente como un sistema donde las interrelaciones se encuentran determinadas por el ámbito socioeconómico/ cultural, contribuyendo a la construcción de nuevos modelos de desarrollo donde se incluye a toda la sociedad en acciones de protección y manejo ambiental, percibiendo el ambiente en última instancia como un objeto que debe ser protegido y conservado (Eschenhagen, 2009).

De allí que resulte pertinente la construcción del seminario de enseñanza ambiental para la Facultad de Ciencia y Tecnología sin desconocer que aquello que se ha abordado en la Universidad Pedagógica Nacional, frente al tema ambiental, ha implicado esfuerzos investigativos que intentan responder a la complejidad de la problemática desde diferentes enfoques. No obstante, el carácter complejo del entorno aporta a la construcción del sujeto maestro que interactúa y forma parte del mismo, por lo que su cuestionamiento es pertinente apuntando a una mirada integral desde la perspectiva humanística, disciplinar y pedagógica. Por tanto, la enseñanza ambiental no se puede reducir a la proposición de soluciones a problemas ambientales dado que fragmentan el conocimiento así como la estructura social (Eschenhagen, 2009).

\section{Fase de indagación: etapa B}

El análisis de discurso se realizó a los diferentes actores que vinculan la Facultad de Ciencia y Tecnología dentro de los cuales se destaca al decano, 5 coordinadores de programa, 3 profesores y 21 estudiantes que abordaron $-\mathrm{y}$ aún lo hacen- el Seminario de Educación Ambiental. Es necesario aclarar que no fue posible entrevistar a 3 estudiantes del seminario de EA ofrecido para el Departamento de Química, dado que no hubo autorización por parte del profesor a cargo. En la tabla 3 se especifican las tendencias predominantes para ambiente, educación ambiental y problemática ambiental, producto del análisis de las entrevistas.

Tabla 3. Caracterización de entrevistas realizadas a actores de la Facultad de Ciencia y Tecnología

\begin{tabular}{|c|c|c|c|c|c|}
\hline Categoría & & Tendencias & $\begin{array}{c}\text { Decanos y coordinadores } \\
\text { (6 entrevistas) }\end{array}$ & $\begin{array}{c}\text { Profesores (3 } \\
\text { entrevistas) }\end{array}$ & $\begin{array}{c}\text { Estudiantes (21 } \\
\text { entrevistas) }\end{array}$ \\
\hline \multirow{8}{*}{$\begin{array}{c}\text { Postura } \\
\text { académica }\end{array}$} & \multirow{3}{*}{ Ambiente } & Objeto & & & 3 \\
\hline & & Sistema & 4 & 2 & 13 \\
\hline & & Crítica a la visión de mundo & & 1 & 5 \\
\hline & \multirow{2}{*}{$\begin{array}{l}\text { Educación } \\
\text { ambiental }\end{array}$} & Acción tecnológica y ciencia aplicada & 6 & 2 & 2 \\
\hline & & Práctica social crítica & & 1 & 16 \\
\hline & \multirow{3}{*}{$\begin{array}{l}\text { Problemática } \\
\text { ambiental }\end{array}$} & Analizar, conocer y solucionar & 4 & 2 & 7 \\
\hline & & Construcción social & 1 & & \\
\hline & & Crisis del conocimiento sobre el mundo & & 1 & 12 \\
\hline $\begin{array}{c}\text { Postura } \\
\text { metodológica }\end{array}$ & \multicolumn{5}{|c|}{$\begin{array}{l}\text { Estrategia al interior del seminario, proyecto de Facultad, reglamentación de la Educación ambiental en la } \\
\text { Universidad. } \\
\text { Componente obligatorio. Seminario para los profesores en formación de la Facultad de Ciencia y Tecnología }\end{array}$} \\
\hline $\begin{array}{c}\text { Postura } \\
\text { investigativa }\end{array}$ & \multicolumn{5}{|c|}{$\begin{array}{l}\text { Contexto y experiencia que incluye al hombre en el ambiente. } \\
\text { La interdisciplinariedad como eje articulador del Seminario de Enseñanza Ambiental } \\
\text { Trabajos de grado como fuente investigativa. } \\
\text { Pertinencia, urgencia y necesidad de éste como electivo (trabajo integral). No solo es el licenciado en biología. }\end{array}$} \\
\hline
\end{tabular}


Como resultado de esta fase de la investigación, se puede afirmar que la visión de educación ambiental, ambiente y problemática ambiental, en la población de estudio de la Facultad de Ciencia y Tecnología, está asociada con la experiencia y el contexto, elementos que constituyen al sujeto como parte del ambiente. Por consiguiente, no se acepta al ambiente como objeto o la suma de relaciones; se percibe como un constructo social mediado por filtros culturales y representaciones simbólicas que están ideológica y políticamente condicionados.

De este modo, se comprende al maestro como una totalidad donde no solo se considera su formación científica o su capacidad para el quehacer, retomando las formas en las que construye conocimiento, su relación con el entorno y con el otro. Así, la enseñanza ambiental no se puede reducir a un sinnúmero de conceptos que se deben conocer; por el contrario, debe partir de su cuestionamiento, debate y reflexión.

En cuanto a educación y problemática ambiental las posturas difieren en las tendencias, pues como se observa en la tabla 3, la mayoría de los profesores en ejercicio reflejan en sus posturas la visión dominante de la educación como herramienta para abordar lo ambiental en el marco de la dinámica económica y científica del mundo moderno, difundida desde las normativas internacionales que intentan promover una forma de tratar el problema ambiental sin ser cuestionado.

Por otra parte, los profesores en formación (estudiantes) en su mayoría cuestionan el origen de lo ambiental desde la vertiente política y económica, por lo que no se trata de intentar solucionar los problemas sino de pensar al hombre como parte de los mismos e integrar la subjetividad en la comprensión de los fenómenos. En otras palabras, es necesario contemplar la incertidumbre del pensamiento y del sujeto, aspectos que según Morin (2007) caracterizan al pensamiento complejo.

Desde la postura metodológica, los actores entrevistados proponen temáticas para la construcción del Seminario de Enseñanza Ambiental, de lo que se destaca el reconocimiento de referentes políticos, económicos, humanísticos, ideológicos, sociales y culturales en los que surge la educación ambiental. Esto hace evidente la preocupación por la formación ambiental recibida, aunque no se busca desconocer el origen y la historia de lo ambiental en la universidad, es necesario plantear una EA alternativa para pensar en una nueva forma de vivir en y con el mundo mediante un proceso de análisis crítico de las realidades ambientales, sociales y educativas con el fin de transformarlas y permitir prácticas discursivas y pedagógicas diferentes al momento de abordarlas en la escuela.

Frente a las estrategias o actividades que se proponen es clara la necesidad de cuestionar, debatir y proponer nuevas formas de incurrir en la formación ambiental del profesor de manera que se plantee una visión interdisciplinar donde cada área desde su forma de conocer el entorno, aporte a la solución de las diferentes problemáticas ambientales y sociales. 
Desde estas posturas se busca la relación del conocimiento de cada disciplina para solventar situaciones concretas evidentes en el entorno, se desconoce que el problema no consiste en el resultado de las formas de actuar sino que se encuentra en las formas de pensar el mundo (Eschenhagen, 2009). Aspecto a tener en cuenta en el desarrollo del Seminario de Enseñanza Ambiental en el cual la mirada interdisciplinar debe superar las barreras impuestas por cada disciplina y converger en el cuestionamiento y la construcción que hacen los sujetos sobre el ambiente.

\section{Fase de caracterización}

Se realizaron observaciones de clase a dos seminarios de educación ambiental propuestos en el periodo 2011-II durante siete sesiones, los cuales se basaron en la misma propuesta de programa académico. Es pertinente mencionar que en ese mismo periodo se ofreció otro seminario para los profesores en formación del Departamento de Química; sin embargo, no se autorizó el ingreso a este, por lo que en la tabla 4 , se presenta el análisis respectivo de los dos seminarios a los que se asistió.

Tabla 4. Categorización de las observaciones de clase a dos de los seminarios de EA ofrecidos durante el segundo periodo académico de 2011 . Convenciones: CyT: ciencia y tecnología

\begin{tabular}{|c|c|c|c|}
\hline & Categorías & $\begin{array}{c}\text { Obligatorio biología } 2011 \text {-ii (7 } \\
\text { sesiones) }\end{array}$ & $\begin{array}{l}\text { Elecivo todo programa Facultad CyT } \\
\qquad 2011-11 \text { (7 sesiones) }\end{array}$ \\
\hline \multirow{3}{*}{$\begin{array}{c}\text { Postura } \\
\text { académica }\end{array}$} & Ambiente & Crítica a la visión de mundo actual & Como sistema \\
\hline & Educación ambiental & Práctica social crítica & Acción tecnológica y ciencia aplicada \\
\hline & Problemática ambiental & Construcción social & Analizar, comprender y solucionar \\
\hline \multicolumn{2}{|c|}{ Postura metodológica } & $\begin{array}{l}\text { Participación activa de los } \\
\text { estudiantes, se da el qué, los } \\
\text { estudiantes proponen el cómo. } \\
\text { Evaluación sobre los discursos de } \\
\text { los estudiantes y lo que proponen } \\
\text { para el abordaje de lo ambiental. }\end{array}$ & $\begin{array}{l}\text { A través de preguntas propuestas, } \\
\text { discusiones, conferencistas de diferentes } \\
\text { instituciones. } \\
\text { Propuestas de EA por parte de los } \\
\text { estudiantes. }\end{array}$ \\
\hline \multicolumn{2}{|c|}{ Postura investigativa } & $\begin{array}{l}\text { Desde la normatividad y la } \\
\text { necesidad de pensar, reflexionar } \\
\text { y cuestionar los planteamientos y } \\
\text { discursos asociados a la EA. }\end{array}$ & $\begin{array}{l}\text { Desde la necesidad de una EA que } \\
\text { responda a las problemáticas que los } \\
\text { estudiantes consideren pertinentes de } \\
\text { abordar. }\end{array}$ \\
\hline
\end{tabular}

Desde la postura académica, metodológica e investigativa de los maestros a cargo de los seminarios analizados, se puede afirmar que están relacionadas directamente con la formación recibida, los discursos construidos y las prácticas asumidas en la experiencia del sujeto. En consecuencia, la forma como se interprete el ambiente, la educación y problemática ambiental van a determinar las estrategias o actividades que proponga el profesor para el desarrollo del seminario.
Para el caso del componente obligatorio del DBI 2011 - -I el ambiente, la EA y la problemática ambiental están asociados a la experiencia del sujeto en y con el entorno, por lo cual, es necesario comprender en primera instancia la dinámica del problema antes de proponer estrategias de solución. Esta visión se refleja en la metodología asumida por el profesor al dar un papel fundamental al discurso y el pensamiento del futuro licenciado en ciencias, dado que él es quién debe proponer formas diferentes de abordar lo ambiental en la escuela. 
Por otra parte, en el componente electivo de la Facultad 2011 -II, las tendencias, se asocian a la aplicación del conocimiento científico en el análisis y solución de problemas ambientales, en la visión sistémica del ambiente, desde las relaciones sociales y biofísicas que se establecen en el marco del desarrollo económico (Eschenhagen, 2009). Esto se evidencia en el proyecto de investigación, en el que se centra cada estudiante, buscando problemas a solucionar desde el campo de conocimiento propio de su disciplina.

Se resalta el abordaje de la historia de la educación ambiental y de algunos documentos como los lineamientos y estándares curriculares que establecen lo que se debe trabajar en torno a la educación ambiental. No obstante, en el Seminario de Enseñanza Ambiental, la reflexión crítica frente a estos documentos debe retomarse dado que de cualquier modo se puede y se debe cuestionar para tomar posiciones diferentes frente a la práctica educativo, particularmente frente a la educación ambiental.

Dentro de esta misma fase de la investigación, se analizaron cuatro programas académicos de EA ofrecidos en la Facultad de Ciencia y Tecnología en diferentes periodos académicos, dos corresponden al seminario obligatorio del Departamento de Biología, uno al seminario obligatorio del Departamento de Química y uno a un electivo de ofrecido por el Departamento de Biología para todos los programas de la Facultad. Es importante aclarar que durante el segundo periodo académico de 2011 se desarrolló un electivo para la facultad en el cual se siguió el mismo programa académico del curso obligatorio del Departamento de Biología. En la tabla 5 se puede observar el resultado del análisis de estos programas en el marco de las categorías seleccionadas en la investigación.

En primera instancia, el hecho de encontrar programas académicos de EA ofrecidos solamente por los departamentos de Biología y Química (tanto obligatorios como electivos) hace evidente la segregación de lo ambiental a las licenciaturas en donde se abordan temas asociados a la naturaleza, es decir, lo verde, la diversidad biológica, y las situaciones a las que se enfrenta (contaminación, deterioro, etc.), reflejando la fragmentación del conocimiento (Eschenhagen, 2009) y de la problemática ambiental en la estructura educativa. Esta situación es preocupante pues se debe recordar que la humanidad en general, independientemente de sus gustos académicos, artísticos etc., depende de los recursos y servicios que ofrece el ambiente; por consiguiente, todo aquello asociado a la problemática ambiental debe ser tema de debate en todos los programas de formación.

En cuanto a la categorización realizada a los programas académicos, se observa que las tendencias de ambiente, EA y problemática ambiental en las cuales se enmarcan, difieren de un programa a otro y reflejan en cierta medida la postura de quienes los propusieron o diseñaron, producto del discurso construido a lo largo de años de formación y trabajo sobre el tema. De igual modo, la postura metodológica e investigativa resulta coherente con la postura 
académica de cada programa en términos de las estrategias que se proponen para abordar lo ambiental en la formación de profesores de ciencias.

Tabla 5. Categorización de 4 programas académicos de EA disponibles en el marco de la investigación. El programa académico del curso obligatorio para el DBI de 2010 a 2011, es el mismo programa del electivo de Facultad ofrecido en 2011 -II.

\begin{tabular}{|c|c|c|c|c|c|c|}
\hline \multicolumn{3}{|c|}{ Categorías } & $\begin{array}{c}\text { Obligatorio de } \\
\text { Biología } 2008 \text { - } \\
20101\end{array}$ & $\begin{array}{l}\text { Obligatorio Biología } \\
2010 \text { II-2011 }\end{array}$ & $\begin{array}{c}\text { Obligatorio } \\
\text { Química } 2011\end{array}$ & $\begin{array}{l}\text { Electivo todo } \\
\text { programa } \\
2010-11\end{array}$ \\
\hline \multirow{8}{*}{ 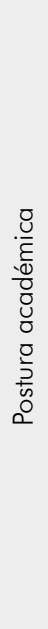 } & \multirow{3}{*}{ Ambiente } & Como Objeto & & & $x$ & \multirow{8}{*}{$\begin{array}{l}\text { No es posible } \\
\text { identificar } \\
\text { tendencias por } \\
\text { falta información }\end{array}$} \\
\hline & & Como Sistema & $x$ & & & \\
\hline & & $\begin{array}{l}\text { Como crítica a la } \\
\text { visión de mundo }\end{array}$ & & $x$ & & \\
\hline & \multirow{2}{*}{$\begin{array}{l}\text { Educación } \\
\text { ambiental }\end{array}$} & $\begin{array}{l}\text { Acción tecnológica } \\
\text { y ciencia aplicada }\end{array}$ & $x$ & & $x$ & \\
\hline & & $\begin{array}{l}\text { Práctica social } \\
\text { crítica }\end{array}$ & & $x$ & & \\
\hline & \multirow{3}{*}{$\begin{array}{l}\text { Problemática } \\
\text { ambiental }\end{array}$} & $\begin{array}{l}\text { Analizar, conocer y } \\
\text { solucionar }\end{array}$ & $x$ & & $x$ & \\
\hline & & Construcción social & & $x$ & & \\
\hline & & $\begin{array}{l}\text { Crisis del } \\
\text { conocimiento sobre } \\
\text { el mundo }\end{array}$ & & & & \\
\hline \multicolumn{3}{|c|}{$\begin{array}{c}\text { Postura } \\
\text { metodológica }\end{array}$} & $\begin{array}{l}\text { Elaboración } \\
\text { de proyectos } \\
\text { ambientales, } \\
\text { participación de } \\
\text { los estudiantes, } \\
\text { construcción de } \\
\text { discurso ambiental. }\end{array}$ & $\begin{array}{l}\text { Se debaten las posturas. } \\
\text { Participación activa de los } \\
\text { estudiantes, construcción } \\
\text { de discurso ambiental a } \\
\text { partir del cuestionamiento } \\
\text { del que ya está. }\end{array}$ & $\begin{array}{c}\text { Desde el } \\
\text { establecimiento } \\
\text { de competencias y } \\
\text { evaluación de las } \\
\text { mismas. }\end{array}$ & $\begin{array}{l}\text { Interacción } \\
\text { profesor } \\
\text { (orientación)- } \\
\text { estudiante } \\
\text { (preparación de } \\
\text { lecturas). }\end{array}$ \\
\hline \multicolumn{3}{|c|}{ Postura investigativa } & $\begin{array}{l}\text { Reflexión sobre } \\
\text { la forma en que } \\
\text { ha sido asumida } \\
\text { la EA desde la } \\
\text { construcción } \\
\text { de criterios y } \\
\text { conocimientos } \\
\text { asociados a esta. }\end{array}$ & $\begin{array}{c}\text { Hacia el futuro profesor } \\
\text { como investigador, } \\
\text { postura crítica frente a los } \\
\text { discursos establecidos. }\end{array}$ & $\begin{array}{c}\text { Desde el discurso } \\
\text { establecido por la } \\
\text { Política Nacional } \\
\text { de Educación } \\
\text { Ambiental (SINA) } \\
\text { Necesidad y } \\
\text { Urgencia por } \\
\text { desarrollo. }\end{array}$ & $\begin{array}{c}\text { Reflexión sobre } \\
\text { los cultivos ilícitos } \\
\text { en el contexto } \\
\text { de los discursos } \\
\text { y las prácticas } \\
\text { de la educación } \\
\text { ambiental }\end{array}$ \\
\hline
\end{tabular}

Haciendo una comparación de los programas se observa que tiende a predominar las visiones de ambiente como objeto y sistema, la EA como acción tecnológica y ciencia aplicada y la problemática como algo para conocer, analizar y solucionar. Esto, además de justificar esta investigación, hace evidente la necesidad de proponer alternativas para abordar lo ambiental de formas diferentes a las ya establecidas en la formación de profe- sores de ciencias. No obstante, los programas académicos analizados constituyen referentes fundamentales para proponer el Seminario de Enseñanza Ambiental, puesto que dan cuenta de cómo se ha abordado lo ambiental en la Facultad de Ciencia y Tecnología, enriqueciendo las discusiones que sobre este tema se han develado en esta investigación, con el fin de aportar a la construcción de discursos sobre lo ambiental en el marco del pensamiento crítico. 


\section{Fase de diseño}

Consiste en dar cuerpo al Seminario de Enseñanza Ambiental para la Facultad de Ciencia y Tecnología, desde los aportes evidenciados en las fases de investigación antes mencionadas. De este modo, el seminario se entiende como un espacio de discusión donde los participantes se encargan de indagar, pensar y cuestionar la información sobre las temáticas a desarrollar. En cuanto a la relación entre el profesor en ejercicio y aquel en formación no se establece de forma unidireccional, dado que el debate es el eje central por el cual los sujetos construyen su conocimiento permanentemente.

Entonces, ofrecer este espacio académico para profesores en formación de ciencias permite reflexionar acerca del surgimiento del ambiente como una construcción humana que se constituye desde la experiencia de cada sujeto, mediado por un conocimiento y una forma de pensar propia de la cultura y un grupo social, donde la complejidad del pensamiento es una alternativa de problematizar permanentemente la educación ambiental desde sus orígenes, referentes, concepciones, tendencias, tipologías, dimensiones, fundamentos y contextos.

De tal forma que el profesor en formación construya y transforme su discurso, que no fragmente o simplifique el problema, sino que lo integre y tenga en cuenta todas sus dimensiones (política, económica y académica) para comprenderlo antes de afirma la existencia de una solución. Asimismo, se asocia el ser y quehacer del sujeto/maestro/investigador, desde el reconocimiento de las posturas académica, investigativa y metodológica, cuestionando cada una de ellas constantemente dándole al seminario la característica de espacio de discusión y debate abierto que apunta a una formación integral, desde una perspectiva humanística, disciplinar y pedagógica, bajo un enfoque transdisciplinar, ya que, busca un intercambio de conocimiento, donde se desdibujan los límites científicos de cada área (Eschenhagen, 2009).

Teniendo en cuenta que el ambiente es un constructo humano, el seminario estaría orientado hacia la enseñanza ambiental, puesto que esta contempla la formación de sujetos, proceso en el cual circulan saberes, experiencias y visiones de mundo que resultan en la configuración de las formas de vivir.

De acuerdo con lo anterior el seminario tiene como objetivo principal contribuir en la formación de los futuros profesores de la Facultad de Ciencia y Tecnología, desde el abordaje crítico de la Educación ambiental teniendo en cuenta sus aspectos teóricos, operativos e investigativos que configuren estrategias de enseñanza ambiental a partir de la complejidad del pensamiento. Para ello se propone la tabla 6, que muestra los tres objetivos específicos y su relación con la metodología del seminario, cuya dinámica está basada en preguntas orientadoras que cuestionan al estudiante así como buscan formas diferentes de abordar la enseñanza ambiental. 
Tabla 6. Relación entre la metodología y los objetivos específicos propuestos para el desarrollo del seminario

\begin{tabular}{l|l}
\hline \multicolumn{1}{c|}{ Objetivos específicos } & \multicolumn{1}{c}{ Estrategias a desarrollar } \\
\hline $\begin{array}{l}\text { Configurar la postura académica del profesor en } \\
\text { formación cuestionando el devenir histórico, normativo } \\
\text { y procedimental de la EA, teniendo como principio de } \\
\text { reflexión la complejidad del pensamiento. }\end{array}$ & $\begin{array}{l}\text { íCómo construye el profesor en formación su discurso ambiental? } \\
\text { elaboración de ensayos que presenta la postura académica del profesor } \\
\text { de esta en la educación). } \\
\text { Maestros, pensemos y discutamos el discurso ambiental: discusiones y } \\
\text { debates permanentes producto de la lectura de documentos asociados } \\
\text { a lo ambiental (normatividades y planteamientos teóricos). }\end{array}$ \\
\hline $\begin{array}{l}\text { Reconocer la postura metodológica del maestro en } \\
\text { formación frente a la enseñanza de lo ambiental, como } \\
\text { fundamento para el cuestionamiento de las formas } \\
\text { en que se ha abordado la educación ambiental en la la } \\
\text { escuela. }\end{array}$ & $\begin{array}{l}\text { clase donde los maestros en formación asuman el espacio académico y } \\
\text { propongan estrategias diferentes de abordar las temáticas del Seminario } \\
\text { de Educación Ambiental. } \\
\text { í cómo va la escuela en relación a la enseñanza ambiental?: visita } \\
\text { a una institución educativa por grupos de trabajo donde se realice una } \\
\text { contextualización en términos del abordaje de lo ambiental, desde los } \\
\text { diversos actores de la institución (estudiantes, maestros, administrativos). }\end{array}$ \\
\hline $\begin{array}{l}\text { Proponer estrategias de enseñanza ambiental a partir } \\
\text { del discurso construido por los maestros en formación } \\
\text { que den cuenta de formas alternativas de relacionar la } \\
\text { escuela con la complejidad de lo ambiental. }\end{array}$ & $\begin{array}{l}\text { Manos a la obra maestros: construcción de una propuesta de } \\
\text { enseñanza ambiental como resultado de discurso construido de la } \\
\text { postura investigativa que los maestros en formación han elaborado } \\
\text { durante el transcurso del seminario. }\end{array}$ \\
\hline
\end{tabular}

En cuanto a la evaluación, en el seminario esta se entiende como un proceso que facilita la toma de decisiones, la reflexión y discusión frente a los contenidos abordados, por lo cual no se asumen conceptos delimitados que se deben transmitir sino que se construyen a la luz de los argumentos y autores que han abordado lo ambiental desde la complejidad, contribuyendo al planteamiento de posturas propias frente al tema ambiental. Así, la evaluación no se refiere únicamente a los contenidos, también se asocia con la reflexión que cada sujeto/maestro en formación realiza frente a su postura académica, metodológica e investigativa.

Así, se proponen 16 sesiones, cada una se centra en una $o$ varias preguntas orientadoras que están asociadas a temáticas como el ambiente, la educación ambiental y la problemática ambiental, su devenir histórico, conceptual, normativo y educativo, para lo cual se proponen lecturas que discuten estos aspectos desde la complejidad del pensamiento.
También se aclaran las actividades a desarrollar y su proceso evaluativo, que se realiza permanentemente tanto de forma cualitativa durante las clases como cuantitativa durante tres cortes planeados para todo el semestre. Por último, se ofrece una bibliografía anexa que puede ser consultada por el maestro en formación, con el fin de complementar el trabajo individual.

\section{Conclusiones}

Desde las revistas y los trabajos de grado realizados en la Facultad de Ciencia y Tecnología de la Universidad Pedagógica Nacional es evidente que la educación ambiental es abordada desde los discursos dominantes al asumirla como una estrategia o metodología para desarrollar objetivos propuestos desde los marcos reglamentarios. Dados estos hallazgos, cobra relevancia la propuesta de tener un acercamiento diferente al tema ambiental desde su enseñanza en el ámbito universitario y más aún en una institución que se proclama así misma educadora de educadores. 
Solamente las revistas y las tesis de los departamentos de Biología y de Química tratan la temática ambiental desde el ámbito educativo, aunque con una fuerte tendencia hacia la educación ambiental como técnica para la solución de problemas, el fomento de actitudes pro ambientales y el ambiente como objeto que es necesario responder a las situaciones que afectan el desarrollo. Justamente de aquí emerge la pertinencia de una propuesta de enseñanza ambiental para todos los programas de la Facultad de Ciencia y Tecnología, donde se dé la opción de discutir los temas asociados a lo ambiental y no solamente de asumirlos.

La forma en la que se asume la problemática ambiental desde los trabajos de grado y revistas, en su gran mayoría se asocian a una visión como problema para solucionar desde aspectos técnicos que se desarrollan con la aplicación del método científico. Además, dichas propuestas, en diversas ocasiones no presentaban una discusión sobre qué es la problemática ambiental y cuáles son sus orígenes sino que se dio por hecho que se debe solucionar un problema particular a través de la educación ambiental. Así, se deja de lado el trasfondo político y económico en el cual se sustenta y surge la problemática como lucha de poderes entre los sujetos y condicionamientos impuestos por un sistema económico legitimado por las mismas dinámicas sociales.

Desde el análisis del discurso se identificó la pertinencia y urgencia de proponer un seminario para toda la Facultad de Ciencia y Tecnología en el cual se tengan en cuenta los objetivos de cada uno de los departamentos, como son la transversalidad y formación e integral; sin embargo se sigue dando una mirada hacia una educación ambiental basada en la construcción de propuestas y estrategias orientadas a la solución de problemas ambientales. En este sentido, frente a la propuesta del Seminario de Enseñanza Ambiental se sugieren temáticas como la historia de la EA, sus conceptos de ambiente y la postura legislativa de Colombia y de la Universidad en particular con respecto a la educación ambiental.

Desde el análisis del discurso se identifica la necesidad de abordar lo ambiental desde un enfoque interdisciplinar; sin embargo, se aclara que es desde el conocimiento científico de cada una de las disciplinas que se debe asumir la problemática ambiental, de allí que el Seminario de Enseñanza Ambiental se encuentre sujeto a la transversalidad, ya que pretende trascender las barreras de cada disciplina para pensar el problema.

En la Facultad de Ciencia y Tecnología de la Universidad Pedagógica Nacional, solo se han desarrollado seminarios de educación ambiental en los departamentos de Biología y de Química, y recientemente, un electivo para todos los programas de la Facultad. No obstante, solo uno de los seminarios del Departamento de Biología aborda la problemática y la educación ambiental teniendo en cuenta su trasfondo político y económico, los demás programas proyectan una visión del problema como algo a lo que se debe dar una solución ya sea desde la aplicación del conocimiento científico o desde la adquisición de hábitos ambientales responsables. 
Es necesario proponer y también discutir los enfoques de los seminarios que abordan lo ambiental a nivel universitario, puesto que es clave que en la formación de los futuros maestros del país, se piensen y se discutan los discursos asociados al ambiente para tomar postura frente al tema y desarrollar el proceso educativo en función a esta.

\section{Referencias bibliográficas}

Calvo, S. y Pérez, J. (2006). La educación ambiental, relato de los orígenes y de su desarrollo internacional. En: El espejismo de la educación ambiental. Madrid: Ediciones Morata.

Caride, J. y Meira, P. (2001). Educación ambiental y desarrollo humano. Barcelona: Editorial Ariel.

Eschenhagen, M. (1998). Evolución del concepto de desarrollo sostenible y su implantación política en Colombia. Revista de Ciencias Administrativas y Ciencias Sociales, 11.

Eschenhagen, M. (2009). Educación ambiental superior en América Latina. Retos epistemológicos y curriculares. 1a. ed. Colombia: Biblioteca Universidad y Ambiente.

Gaudiano, E. (2001). Otra lectura a la historia de la educación ambiental en América Latina y el Caribe. Revista Doctorado en Medio Ambiente y Desarrollo, 3. México: UFPR.

Gutiérrez, J. (1995a). El medio ambiente perspectiva histórica y conceptualización actual. En: La educación ambiental. Fundamentos Teóricos, propuesta de transversalidad y orientaciones extracurriculares. Pp. Xx-xx Madrid: Ed. Muralla

Gutiérrez, J. (1995b). La educación ambiental hoy: transversalidad curricular y transversalidad institucional. En: La educación ambiental. Fundamentos Teóricos, propuesta de transversalidad y orientaciones extracurriculares. Pp. Xx-xx. Madrid: Ed. Muralla.

Leff, E. (2004). Racionalidad ambiental. La reapropiación social de la naturaleza. Buenos Aires: Siglo XXI Editores.

Leff, E. (2007). La complejidad ambiental. Revista Virtual Gaia Scientia 1 (1).

Maya, A. (2009). Poeta-Filósofo del Pensamiento Ambiental Latinoamericano. Sección Filosofía Ambiental Sudamericana, 6. Bogotá: ISEE.

Morin, E. (2007). Introducción al pensamiento complejo. Barcelona: Editorial Gedisa.

Rojas, E. (2006). Antecedentes de una propuesta: hacia la educación ambiental. [En línea]. http:// lunazul.ucaldas.edu.co

Sauvé, L. (1994). "Exploración de la diversidad de conceptos y de prácticas en la educación relativa al ambiente" En: Seminario Internacional: La dimensión ambiental y la escuela. Memorias. Ministerio de Educación Nacional: Bogotá.

SINA (2002). Política Nacional de Educación Ambiental. Bogotá: Ministerio del Medio Ambiente, Ministerio de Educación.

Tréllez, E. (2006). Algunos elementos del proceso de construcción de la Educación Ambiental en América Latina. Revista Iberoamericana de Educación, 41. Asociación Cultural Pirámide. 